\title{
Neural Correlates of Three Promising Endophenotypes of Depression: Evidence from the EMBARC Study
}

\author{
Christian A Webb', Daniel G Dillon', Pia Pechtel', Franziska K Goer', Laura Murray', Quentin JM Huys ${ }^{2,3}$, \\ Maurizio Fava ${ }^{4}$, Patrick J McGrath ${ }^{5}$, Myrna Weissman ${ }^{5}$, Ramin Parsey ${ }^{6}$, Benji T Kurian ${ }^{7}$, Phillip Adams ${ }^{5}$, \\ Sarah Weyandt ${ }^{7}$, Joseph M Trombello ${ }^{7}$, Bruce Grannemann ${ }^{7}$, Crystal M Cooper ${ }^{7}$, Patricia Deldin ${ }^{8}$, \\ Craig Tenke ${ }^{5}$, Madhukar Trivedi ${ }^{7}$, Gerard Bruder ${ }^{5}$ and Diego A Pizzagalli*, \\ 'Department of Psychiatry, Harvard Medical School and McLean Hospital, Belmont, MA, USA; '2Centre for Addiction Disorders, Department of \\ Psychiatry, Psychotherapy and Psychosomatics, Hospital of Psychiatry, University of Zurich, Switzerland; ${ }^{3}$ Translational Neuromodeling Unit, Institute \\ for Biomedical Engineering, University of Zurich and Swiss Federal Institute of Technology (ETH) Zurich, Switzerland; ${ }^{4}$ Clinical Research Program, \\ Massachusetts General Hospital, Boston, MA, USA; ${ }^{5}$ Department of Psychiatry, New York State Psychiatric Institute, College of Physicians and \\ Surgeons of Columbia University, New York, NY, USA; 'Department of Psychiatry and Behavioral Science, Stony Brook University, Stony Brook, NY, \\ USA; ${ }^{7}$ Department of Psychiatry, University of Texas Southwestern Medical Center, Dallas, TX, USA; ${ }^{8}$ Department of Psychiatry, University of \\ Michigan Health System, Ann Arbor, MI, USA
}

\begin{abstract}
Major depressive disorder (MDD) is clinically, and likely pathophysiologically, heterogeneous. A potentially fruitful approach to parsing this heterogeneity is to focus on promising endophenotypes. Guided by the NIMH Research Domain Criteria initiative, we used source localization of scalp-recorded EEG resting data to examine the neural correlates of three emerging endophenotypes of depression: neuroticism, blunted reward learning, and cognitive control deficits. Data were drawn from the ongoing multi-site EMBARC study. We estimated intracranial current density for standard EEG frequency bands in 82 unmedicated adults with MDD, using Low-Resolution Brain Electromagnetic Tomography. Region-of-interest and whole-brain analyses tested associations between resting state EEG current density and endophenotypes of interest. Neuroticism was associated with increased resting gamma (36.5-44 Hz) current density in the ventral (subgenual) anterior cingulate cortex (ACC) and orbitofrontal cortex (OFC). In contrast, reduced cognitive control correlated with decreased gamma activity in the left dorsolateral prefrontal cortex (dIPFC), decreased theta $(6.5-8 \mathrm{~Hz})$ and alpha2 $(10.5-12 \mathrm{~Hz})$ activity in the dorsal ACC, and increased alpha2 activity in the right dIPFC. Finally, blunted reward learning correlated with lower OFC and left dIPFC gamma activity. Computational modeling of trial-by-trial reinforcement learning further indicated that lower OFC gamma activity was linked to reduced reward sensitivity. Three putative endophenotypes of depression were found to have partially dissociable resting intracranial EEG correlates, reflecting different underlying neural dysfunctions. Overall, these findings highlight the need to parse the heterogeneity of MDD by focusing on promising endophenotypes linked to specific pathophysiological abnormalities.

Neuropsychopharmacology (20 I 6) 4I, 454-463; doi:I 0. I 038/npp.20 I 5. I65; published online I July 20 I5
\end{abstract}

\section{INTRODUCTION}

Major depressive disorder (MDD), as currently defined, represents a highly heterogeneous disorder that is etiologically and pathophysiologically complex. One potentially promising approach to parsing this heterogeneity is the investigation of endophenotypes (Chan and Gottesman, 2008; Gottesman and Gould, 2003). Endophenotypes are assumed to lie on the pathway between genotype and disease, and be less complex than the downstream symptom clusters that currently define psychiatric diagnoses. Gottesman and

*Correspondence: Dr DA Pizzagalli, Center for Depression, Anxiety, and Stress Research, McLean Hospital, Harvard Medical School, I I 5 Mill Street, Belmont, MA 02478, USA, Tel: + 617855 4230, Fax: + 617 855 4230, E-mail: dap@mclean.harvard.edu

Received 16 February 2015; revised 24 April 2015; accepted 27 May 2015; accepted article preview online 12 June 2015 colleagues proposed that endophenotypes must meet the following criteria: (1) be associated with the disease; (2) be heritable; (3) be primarily state-independent; (4) cosegregate within families; (5) be more common in non-affected family members of individuals with the disease compared with the general population; and (6) be measured reliably.

Neuroticism, blunted reward learning and cognitive control deficits have emerged as among the most promising behavioral endophenotypes of depression (Goldstein and Klein, 2014; Pizzagalli, 2014; Snyder, 2013), and map well onto central Research Domain Criteria (RDoC) domains, particularly negative valence systems (neuroticism), positive valence systems (reward learning), and cognitive systems (cognitive control). First, neuroticism-the propensity to experience negative emotions-is elevated in current and remitted depression (De Fruyt et al, 2006; Klein et al, 2011), and predicts first onset of MDD (Kendler et al, 2006). Twin 
studies indicate that neuroticism shares significant genetic variance with depression, and is moderately heritable (eg, Birley et al, 2006; Kendler et al, 2006). Moreover, neuroticism cosegregates within families (Farmer et al, 2002; Ouimette et al, 1996), and non-depressed family members of those with MDD have higher levels of neuroticism than the general population (Modell et al, 2003). Finally, measures of neuroticism (eg, NEO Personality Inventory) have relatively strong psychometric properties (Costa et al, 2005).

Second, blunted reward learning-defined as a diminished ability to modulate behavior as a function of rewards-has been observed in both individuals with current and remitted MDD (Goldstein and Klein, 2014; Pechtel et al, 2013; Pizzagalli, 2014). A twin study estimated the heritability of reward learning to be 0.46 (Bogdan and Pizzagalli, 2009), as assessed by a commonly used Probabilistic Reward Task (PRT; Pizzagalli et al, 2005a). In addition, the same study reported a moderate $(r=0.29)$ genetic correlation between depression and reward learning. Data related to the reliability of reward learning is limited, with one study finding significant test-retest reliability $(r=0.57)$ over 38 days (Pizzagalli et al, 2005a). To our knowledge, no study has evaluated whether reward learning fulfills the cosegregation or familial association endophenotype criteria.

Finally, MDD is characterized by broad impairments in cognitive control, which often persist following remission (Snyder, 2013) and exhibit trait-like stability (Sarapas et al, 2012). Moderate-to-high heritability (Friedman et al, 2008; Stins et al, 2004), as well as strong test-retest reliability and internal consistency (Wöstmann et al, 2013) have been reported for commonly used cognitive control paradigms (eg, Stroop, Eriksen Flanker). In addition, there is evidence of cognitive control impairments in healthy, unaffected twins discordant for MDD (Christensen et al, 2006). Evidence for cosegregation and familial association is lacking.

With regards to neural substrates, neuroticism has been associated with increased amygdala and anterior cingulate cortex (ACC) activity (Haas et al, 2007; Servaas et al, 2013), both in the 'affective' (ie, rostral (rACC) and subgenual (sgACC)) and 'cognitive' subdivisions (ie, dorsal (dACC)). In contrast, tasks tapping reward learning have been shown to recruit the striatum, orbitofrontal cortex (OFC; Hornak et al, 2004; O’Doherty, 2004), dACC (Rushworth et al, 2007; Santesso et al, 2008), and left dorsolateral prefrontal cortex (dlPFC; BA 9/46; Ahn et al, 2013; Pizzagalli et al, 2005b), especially in tasks requiring integration of reinforcements over time (eg, PRT). Finally, cognitive control has been reliably linked to the dlPFC and dACC (Pizzagalli, 2011; Ridderinkhof et al, 2004).

Inspired by the RDoC initiative, the goal of this study was to examine the association between these three candidate endophenotypes and intracranial estimates of resting brain electrical activity (EEG) in an unmedicated depressed sample. We employed a region-of-interest (ROI) approach, followed by an exploratory whole-brain analysis to test the specificity of ROI findings. ROIs were selected based on the literature reviewed above, and within the constraints of EEG source localization techniques, which cannot probe subcortical structures (eg, amygdala, caudate). Based on prior studies, we hypothesized that: (1) neuroticism would be associated with greater resting activity in the affective (rACC/sgACC) and cognitive (dACC) subdivisions of the ACC; (2) blunted reward learning would be linked to lower activity in the OFC, dACC, and left dlPFC; and (3) reduced cognitive control would correlate with lower activity in the dlPFC and cognitive (dACC)-but not affective-ACC subdivision. Finally, a cluster analysis was conducted in an effort to empirically derive subgroups of depressed patients on the basis of endophenotype profiles.

\section{MATERIALS AND METHODS}

Data were collected in a multi-site clinical trial entitled 'Establishing Moderators and Biosignatures of Antidepressant Response for Clinical Care for Depression' (EMBARC). Recruiting sites are Columbia University (CU), Massachusetts General Hospital (MGH), the University of Texas Southwestern Medical Center (UT), and the University of Michigan (UM). Participants completed several behavioral, self-report, and physiological assessments before enrolling in the double-blind, placebo-controlled clinical trial of sertraline and bupropion. Data collection for the treatment phase is ongoing and the blind remains unbroken. Accordingly, the current study only considers baseline resting EEG, behavioral (Flanker and PRT), and self-report (NEO) data for the first 100 MDD subjects. Participants provided informed consent following procedures approved by site IRBs.

\section{Participants}

Eligible participants (aged 18-65 years) met DSM-IV criteria for a current MDD episode (SCID-I/P), scored 14 or above on the 16-item Quick Inventory of Depression Symptomatology (Rush et al, 2003), and were medication free for $>3$ weeks before completing any study measures. Exclusion criteria included: history of psychosis or bipolar disorder; substance dependence in the past 6 months (excluding nicotine) or substance abuse in the past 2 months; active suicidality; or unstable medical conditions (for more information about the sample, see Supplementary Materials). Data from $82 \mathrm{MDD}$ subjects who passed quality control criteria for both Flanker and PRT (see Supplementary Materials) and had NEO scores were analyzed.

\section{Measures}

NEO Five-Factor Inventory: 3 (NEO-FFI-3). The NEOFFI is a 60 -item self-report questionnaire assessing Neuroticism, Extraversion, Openness, Agreeableness and Conscientiousness (12 items/factor; McCrae and Costa, 2010).

Probabilistic Reward Task (PRT). The PRT uses a differential reinforcement schedule to probe reward learning (ie, the ability to modulate behavior as a function of rewards), and has been described in detail (Pizzagalli et al, 2005a, 2008; see Supplementary Materials). Participants performed two blocks of 100 trials.

Flanker Task. A modified version of the Eriksen Flanker Task with an individually titrated response window was used (Eriksen and Eriksen, 1974; Holmes et al, 2010). Participants first completed a practice session followed by five blocks consisting of 70 trials each (46 congruent and 24 incongruent), for a total of 350 trials. On each trial, participants 
pressed a button to indicate whether a center arrow pointed left or right. The central arrow was presented with adjacent flankers, which either pointed in the same direction (congruent condition) or the opposite direction (incongruent condition) as the central arrow (see Supplementary Materials). Both accuracy and reaction time (RT) were recorded.

Hamilton Rating Scale for Depression (HRSD). The 32item HRSD is a clinician-administered measure of depressive symptom severity (Hamilton, 1960). It was administered by trained clinical evaluators.

\section{Data Acquisition and Reduction}

Resting EEG recording consisted of four contiguous 2-min trials (two eyes open, two eyes closed), for a total of $8 \mathrm{~min}$. Site certification, intersite standardization, quality assurance, and preprocessing of resting EEG for all sites were performed at the Columbia site (see Supplementary Materials for details). McLean Hospital was responsible for site training and certification, quality assurance, and analysis of Flanker and PRT data as well as Low-Resolution Brain Electromagnetic Tomography (LORETA) analyses. Hypotheses were tested for the theta $(6.5-8 \mathrm{~Hz})$, alpha1 $(8.5-10 \mathrm{~Hz})$, alpha2 $(10.5-12 \mathrm{~Hz}$ ), and gamma $(36.5-44 \mathrm{~Hz})$ frequency bands (for rationale, see Supplementary Materials). LORETA (PascualMarqui et al, 1999) was used to estimate the threedimensional intracerebral current density distribution for each frequency band. With respect to the ACC, to test the specificity of findings, LORETA voxels corresponding to the affective (BA25 (sgACC); BA24/32 (rACC)) and cognitive (BA24'/32' (dACC)) subdivisions were identified (Pizzagalli, 2011). Similarly, anatomical considerations were used to identify the dlPFC (lateral BA9 and BA46) and OFC (BA11/13/47/12; Kringelbach and Rolls, 2004; see Supplementary Material, Supplementary Figure 1, for ROI definitions). For each ROI, intensity-normalized current density was averaged across voxels.

Probabilistic Reward Task. The primary variable of interest was change in response bias (RB) scores from the first to the second block $\left(\mathrm{RB}_{\text {Block2 } 2}-\mathrm{RB}_{\text {Block1 } 1}\right)$, which reflects reward learning. In addition, discriminability scores, indexing the ability to differentiate between stimuli, were included as a covariate in specificity analyses (for formulas, see Supplementary Materials). The development of a RB depends both on the sensitivity to individual rewarding feedback and the ability to incrementally associate stimuli/actions with rewards over the course of the task. To discriminate between these components of reward learning, we fitted a series of reinforcement learning models to trial-by-trial behavioral data as described previously (Huys et al, 2013). These contained two key parameters: reward sensitivity, which indexes the reinforcing strength of the individual reward outcomes, and learning rate, which measures the ability to progressively associate outcomes with antecedent stimuli/ actions (see Supplementary Materials).

Flanker task. The primary variables of interest were the interference effects: lower accuracy and longer RTs on incongruent than congruent trials. These effects were com- puted as (Accuracy Compatible trials $_{\text {- Accuracy }}$ Incompatible trials) and $\left(\mathrm{RT}_{\text {Incompatible trials }}-\mathrm{RT}_{\text {Compatible trials }}\right)$.

\section{Statistical Analyses}

For ROI-based analyses, four separate sets of multiple regressions were run to examine the association between LORETA-derived EEG current density and (1) neuroticism, (2) reward learning, and the Flanker interference effects on both (3) accuracy and (4) RT. For neuroticism, current density in the affective (sgACC and rACC) and cognitive (dACC) subdivisions of the ACC were entered as predictors. For reward learning, current density in the OFC, left and right dIFPC, and dACC were entered as predictors. Finally, for Flanker interference effects, separate multiple regressions were run for accuracy and RT, with current density in the rACC, dACC, and left/right dlPFC serving as predictors. Regressions were run separately for each frequency band. All predictor variables were entered simultaneously in multiple regression models, and all models included site (CU/MGH/ UT/UM) as a covariate. For significant findings, specificity analyses were performed to test whether associations remained when accounting for (1) depression severity (by entering HRSD scores as an additional predictor) and (2) the other endophenotypes. In addition, to the extent that significant findings emerged in the reward learning analyses, we followed-up with two multiple regressions testing whether significant ROIs also predicted the reward sensitivity and learning rate parameters extracted from the computational models. Regression analyses were carried out using SAS version 9.2 PROC GLM. [Several univariate outliers (absolute value $z>3.0$ ) were identified. Specifically, gamma, theta, alpha1, and alpha2 current density values in BA24/32 (rACC) for 1 subject; theta, alpha1 and alpha2 in BA24'/32' (dACC) for 1 subject; gamma and alphal left dlPFC values for 1 subject. These data were omitted from the relevant analyses.]

To evaluate regional specificity, exploratory whole-brain correlational analyses were conducted between (1) neuroticism, reward learning and Flanker interference effects, and (2) current density in the theta, alpha1, alpha2, and gamma bands separately. To control for multiple comparisons, analyses were carried out using the 'Randomise' permutationbased inference tool in FSL for nonparametric statistical thresholding (5000 permutations, $p<0.05$, FWE corrected; Winkler et al, 2014). Finally, in an effort to empirically derive subgroups of depressed patients on the basis of endophenotype measures, a two-step cluster analysis was conducted in SPSS (Version 20) using the Log-likelihood distance measure and Schwarz's Bayesian Information Criterion as clustering criteria (Chiu et al, 2001; Fava et al, 2012), and without prespecifying the desired number of clusters.

\section{RESULTS}

Supplementary Table 1 provides a summary of significant ROI findings.

\section{Neuroticism}

The first regression predicted neuroticism scores, with gamma current density in the cognitive (dACC) and affective (sgACC) ACC subdivisions entered as predictors. As 


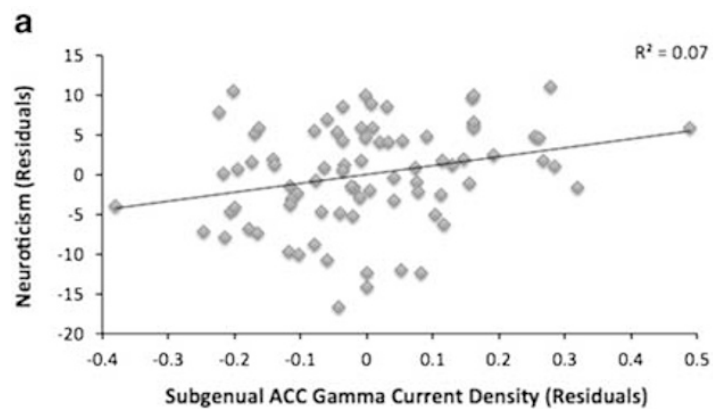

b
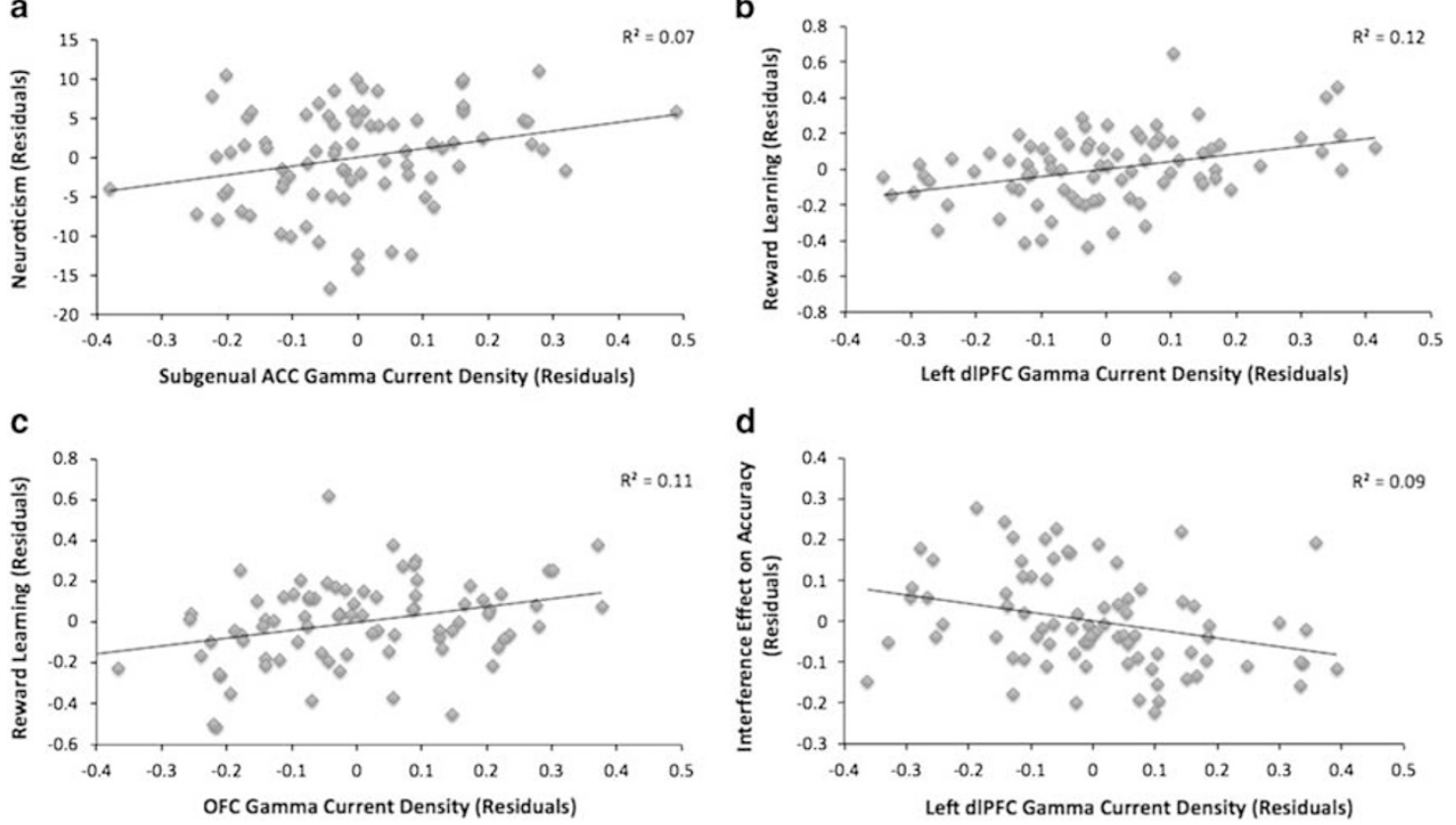

Figure I Partial regression plots displaying the association between (a) neuroticism and gamma current density in the subgenual anterior cingulate cortex (sgACC; BA25), after controlling for the model covariates (site and gamma activity in BA 24'/32' (dACC)); (b) reward learning and gamma activity in the left dorsolateral prefrontal cortex (dIPFC), after controlling for the model covariates (site and gamma activity in the right dIPFC, dACC, and orbitofrontal cortex (OFC)); (c) reward learning and gamma activity in the OFC, after controlling for the model covariates (site and gamma activity in the left and right dIPFC, and $\mathrm{dACC}$ ); (d) Flanker interference effect on accuracy and gamma activity in the left dIPFC, after controlling for the model covariates (site and gamma activity in the right dIPFC, $d A C C$, and BA 24/32 (rACC)). The association in panel a remains significant $(\beta=0.29, p=0.030)$ after removing the sgACC residual value at the far right of panel.

expected, when controlling for dACC, gamma activity in sgACC was associated with neuroticism $(\beta=0.31, p=0.018$; Figure 1a). In contrast, gamma activity in dACC did not predict neuroticism $(\beta=-0.01, p=0.965)$. An analogous model entering gamma current density in the dACC and rACC yielded a nonsignificant trend between rACC and neuroticism $(\beta=0.24, p=0.054$; dACC: $\beta=-0.07$, $p=0.646)$. [Given the very high correlation between current density in BA25 (sgACC) and neighboring BA24/32 (rACC) (for gamma, $r=0.74$ ), these variables were included as predictors in separate regression models to minimize multicollinearity.] No significant predictors emerged for the theta or alpha bands $(p$ 's $>0.15)$.

Specificity analyses. The relationship between sgACC gamma activity and neuroticism remained when accounting for (1) current depressive symptoms (HRSD) and the other NEO personality factors $(\beta=0.27, p=0.024)$; and (2) Flanker interference effects and reward learning $(\beta=0.29, p=0.025)$.

\section{Reward Learning}

Greater gamma activity in the OFC $(\beta=0.33, p=0.004)$ and left dlPFC $(\beta=0.34, p=0.002)$-but not right dlPFC $(\beta=$ $-0.11, p=0.358)$ or dACC $(\beta=-0.05, p=0.682)$-predicted reward learning (Figure $1 \mathrm{~b}$ and $\mathrm{c}$ ). Importantly, the correlations between reward learning and gamma activity in the left $(r=0.31, p=0.005)$ and right dlPFC $(r=-0.04, p=0.711)$ were significantly different $(z=2.36, p=0.018$; Meng et al, 1992). Models considering theta and alpha current density yielded no significant associations ( $p$ 's $>0.06)$.
Specificity analysis. The relationships between gamma activity in the OFC/left dlPFC and reward learning remained significant when accounting for (1) current depressive symptoms and PRT discriminability (both $\beta$ 's $=0.34, p<0.005$ ); and (2) neuroticism and the Flanker interference effects (OFC: $\beta=0.33, p=0.008$; left dlPFC: $\beta=0.41, p<0.001$ ).

Computational modeling. A multiple regression indicated that gamma activity in the OFC $(\beta=0.27, p=0.043)$-but not the left dlPFC $(\beta=0.11, p=0.378)$ - positively predicted reward sensitivity. No significant findings emerged in the prediction of learning rate $(p$ 's $>0.20)$.

\section{Cognitive Control}

Flanker interference effect (accuracy). The regression revealed that gamma activity in the left $\operatorname{dlPFC}(\beta=-0.31$, $p=0.009)$-but not right dlPFC $(\beta=0.03)$, dACC $(\beta=0.20)$, or $\operatorname{rACC}(\beta=-0.05)$ (all $p$ 's $>0.15)$ - was a significant negative predictor of the Flanker interference accuracy effect (Figure 1d). The correlations involving the left $(r=-0.30$, $p=0.007)$ and right $(r=-0.04, p=0.71)$ dlPFC gamma activity were different only at a trend level $(z=-1.72$, $p=0.085)$. Models for theta and alpha yielded no effects $(p$ 's $>0.05)$.

Specificity analysis. The relationship between gamma current density in the left dlPFC and the Flanker interference effect was confirmed when accounting for (1) current depressive symptoms $(\beta=-0.32, p<0.009)$ and $(2)$ neuroticism and reward learning $(\beta=-0.30, p=0.020)$. 

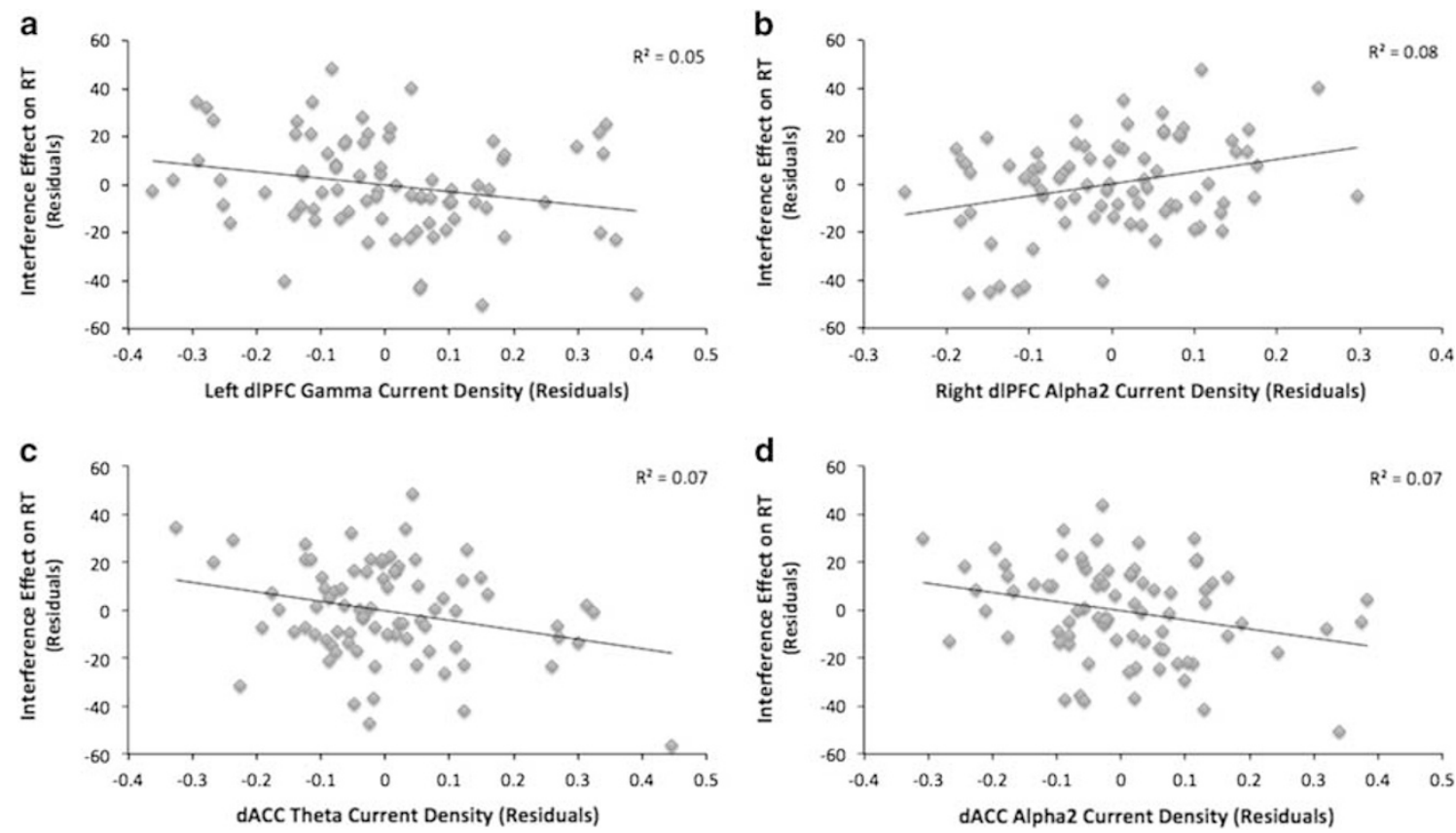

Figure 2 Partial regression plots displaying the association between Flanker interference effect on reaction time (RT) and (a) gamma current density in left dIPFC, after controlling for model covariates (site and gamma activity in the right dIPFC, BA 24'/32' (dACC) and BA 24/32 (rACC), (b) alpha2 current density in the right dIPFC, controlling for site and alpha2 activity in the left dIPFC, dACC, and rACC, (c) theta current density in dACC, controlling for theta activity in rACC, and left and right dIPFC, and (d) alpha2 current density in AACC, controlling for alpha2 activity in rACC, and left and right dIPFC. The theta dACC effect is reduced to a trend level $(\beta=-0.29, p=0.060)$ in the model after removing the residual value at the bottom right corner of panel $c$. It should be noted that the zero-order correlation (ie, excluding model covariates) between theta dACC and interference effect on RT is significant $(r=-0.25 ; p=0.026)$.

Table I Whole-Brain Correlations between Candidate Endophenotypes and LORETA-Estimated Current Density

\begin{tabular}{|c|c|c|c|c|c|c|}
\hline \multirow[b]{2}{*}{ Covariate } & \multirow[b]{2}{*}{ Region (frequency band) } & \multirow[b]{2}{*}{ Number of voxels } & \multicolumn{3}{|c|}{ Peak-r MNI coordinates } & \multirow[b]{2}{*}{ Mean $r$} \\
\hline & & & $x$ & $y$ & $\mathbf{z}$ & \\
\hline Reward learning & $\mathrm{L} \operatorname{dIPFC/OFC~}(\gamma)$ & 51 & -38 & 52 & 8 & $0.38 * * * *$ \\
\hline Flanker ACC & $\mathrm{L} \operatorname{dIPFC}(\gamma)$ & 4 & -38 & 38 & 36 & $-0.38 * * * *$ \\
\hline
\end{tabular}

Abbreviations: dIPFC, dorsolateral prefrontal cortex; OFC, orbitofrontal cortex; sgACC, subgenual anterior cingulate cortex; $\gamma$, Gamma $(36.5-44 \mathrm{~Hz})$.

For each cluster, the number of voxels exceeding the statistical threshold is reported (using the 'Randomise' permutation-based inference tool in FSL for nonparametric statistical thresholding, with 5000 permutations, $p<0.05$, FWE corrected for multiple comparisons; Winkler et al, 20 I4); Neuroticism = NEO Five-Factor Inventory (Neuroticism total score); Reward Leaming = Change in response bias scores from block I to block 2 in the Probabilistic Reward Task; Flanker ACC = Flanker interference effect on accuracy. Peak$r \mathrm{MNI}$ coordinates = MNI coordinates for the voxel associated with the largest Pearson's correlation between the covariate and current density (unit: amperes per square meter, $\mathrm{A} / \mathrm{m}^{2}$ ); Mean $r=$ the mean Pearson's $r$ averaged across all voxels belonging to the cluster. Similar to the analyses above, site is included as a covariate.

$* p<0.05$, $* * * * 0.00$ I (uncorrected).

Flanker interference effect $(R T)$. When considering gamma, only current density in the left dlPFC was a negative predictor of interference RT effects $(\beta=-0.23, p=0.045$; Figure 2a). For theta, only dACC activity was negatively associated with the interference RT effect $(\beta=-0.33$, $p=0.019$; Figure 2c). For alpha2, dACC activity was negatively associated $(\beta=-0.31, p=0.020)$ and right dlPFC activity positively associated $(\beta=0.51, p=0.012)$, with the interference effect (Figure $2 \mathrm{~b}$ and $\mathrm{d}$ ). All other predictors were nonsignificant $(p$ 's $>0.05)$.

Specificity analysis. The above relationships were confirmed when accounting for (1) current depressive symptoms (theta dACC: $\beta=-0.34, p=0.018$; alpha2 dACC: $\beta=-0.34, p=0.012$; alpha2 right dlPFC: $\beta=0.58, p=0.005$ ), with the exception of gamma activity in the left dlPFC $(\beta=-0.22, p=0.054)$, and (2) neuroticism and reward learning (theta dACC: $\beta=-0.32$; gamma left dlPFC: $\beta=-0.32$; alpha2 right dlPFC: $\beta=0.58$; alpha2 dACC: $\beta=-0.34, p^{\prime} s<0.020$ ).

\section{Exploratory Whole-Brain Analyses to Examine Specificity of Findings}

For the whole-brain correlations with neuroticism, 36 voxels spanning the bilateral OFC and sgACC/rACC (BA11/47, BA25, BA24) correlated positively with gamma activity 


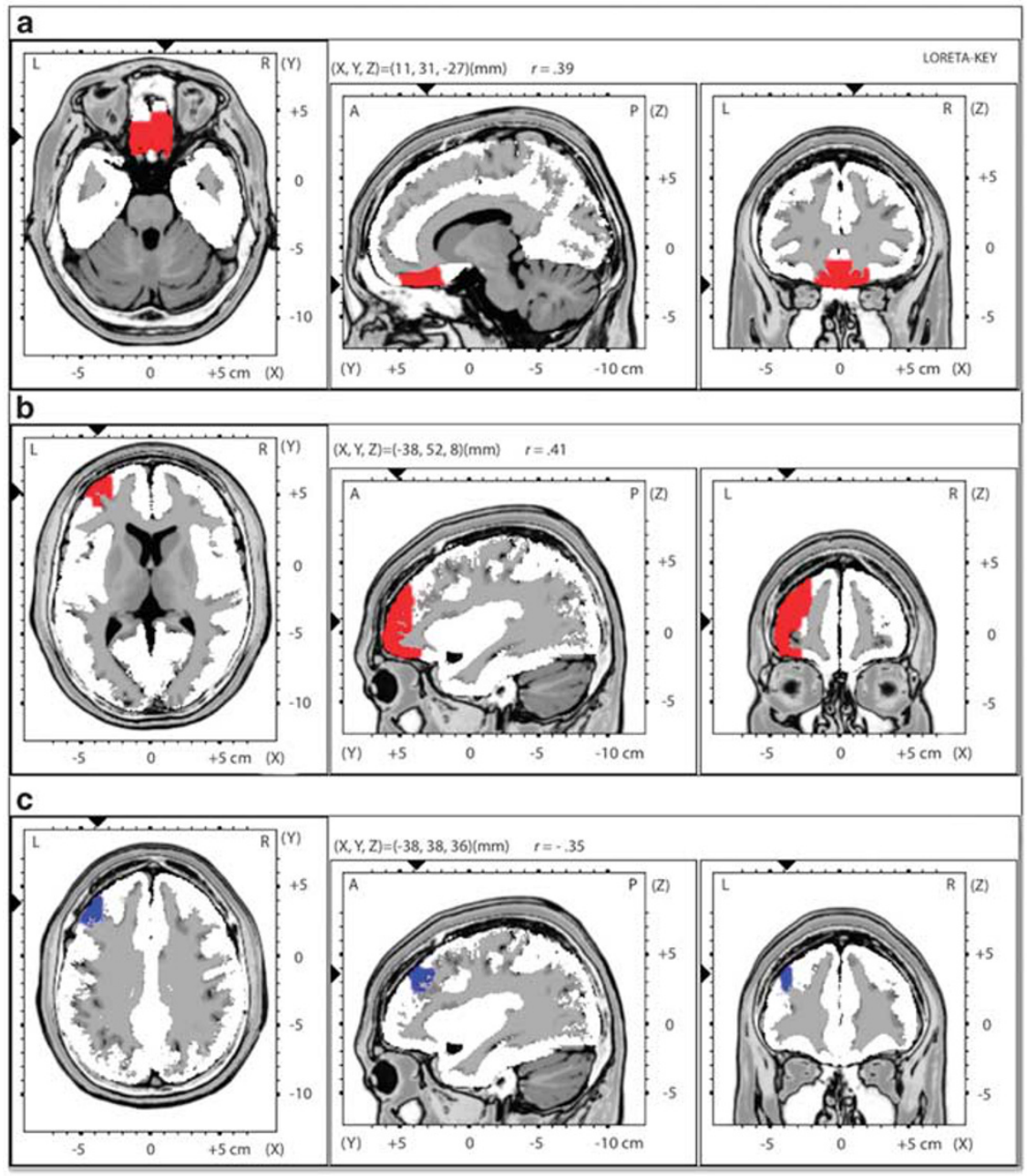

Figure 3 Whole-brain analysis displaying voxel-by-voxel correlations between (a) gamma $(36.5-44 \mathrm{~Hz}$ ) current density and neuroticism (displaying axial/ sagittal/coronal views of the bilateral OFC/subgenual ACC cluster (36 voxels) that was significantly positively correlated with neuroticism); (b) gamma current density and reward learning (displaying axial/sagittal/coronal views of the left dIPFC/OFC cluster (5I voxels) that was significantly positively correlated with reward learning); (c) gamma current density and Flanker interference effect on accuracy (displaying axial/sagittal/coronal views of the left dIPFC cluster (4 voxels) that was significantly negatively correlated with the compatibility effect on accuracy).

(Table 1 and Figure 3a). For reward learning, 51 voxels encompassing the left OFC to the dlPFC (including lateral BA10, BA11, BA47, BA9 and BA46) correlated positively with gamma activity (Table 1 and Figure $3 b$ ). For the Flanker accuracy interference effect, 4 voxels were significantly negatively correlated with gamma activity (left dlPFC; BA9/10; Table 1 and Figure 3c). For the interference RT effect, no significant effects emerged.

\section{Intercorrelations between Neuroticism, Reward Learning, and Flanker Performance}

The three endophenotypes were not significantly intercorrelated (see Supplementary Material).

\section{Cluster Analysis}

In an effort to delineate subgroups of depressed patients on the basis of endophenotype profiles, we entered the following standardized variables into a two-step cluster analysis: neuroticism, PRT reward learning scores, and the Flanker accuracy interference effect. A three-cluster solution yielded the best fit (Bayesian Information Criterion $=180.69$ and $\mathrm{LL}=1.21)$. The first cluster included $41 \%(N=34)$ of the sample, the second cluster $29 \%(N=24)$, and the third cluster $28 \%(N=23)$. ANOVAs revealed significant differences across clusters in neuroticism $(\mathrm{F}(2,78)=52.39, p<0.001)$, reward learning $(\mathrm{F}(2,78)=75.41, p<0.001)$, and the interference effect $(\mathrm{F}(2,78)=11.46, p<0.001)$. Notably, there were no significant differences across clusters in total HRSD depression scores $(\mathrm{F}(2,78)=1.32, p=0.27$; see Figure 4 for additional details).

\section{DISCUSSION}

MDD is increasingly recognized as a highly heterogeneous disorder both in terms of symptom presentation and etiology/pathophysiology. To parse this heterogeneity, there has been increased interest in examining relatively less complex and more tractable endophenotypes. The present study examined whether three promising endophenotypes of 
a

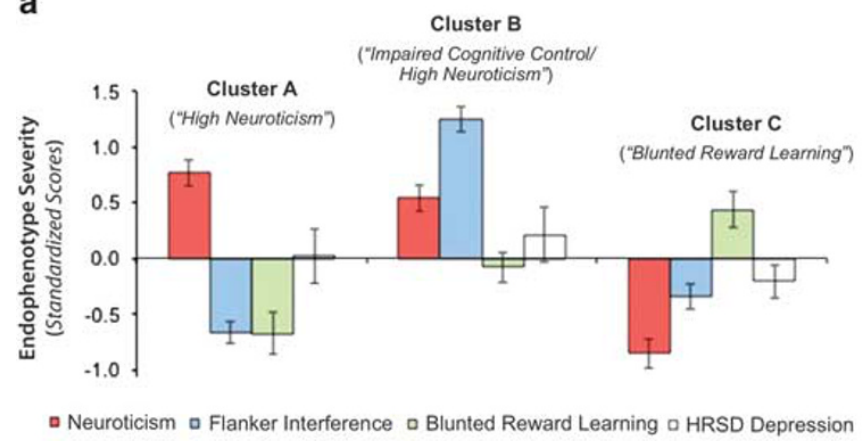

b

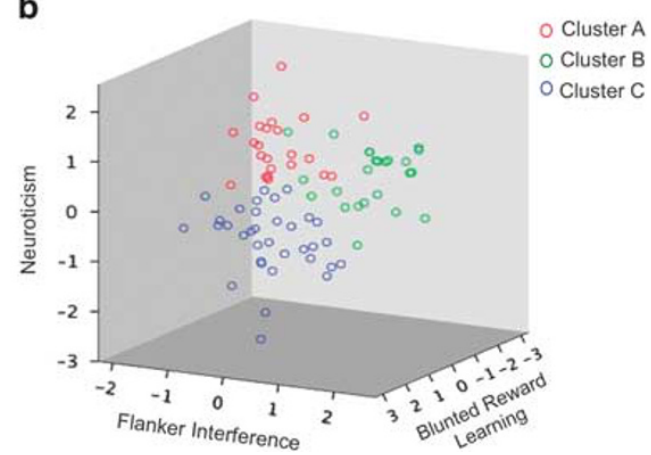

Figure 4 (a) Standardized means (with standard error bars) are displayed for neuroticism (NEO), Flanker interference effect on accuracy, blunted reward learning (PRT), and total depression (HRSD) scores for each of three groups derived from the two-step cluster analysis. (b) Three-dimensional scatterplot displaying association between the three endophenotype variables by cluster group. For consistency across measures, the PRT reward learning variable was reverse scored such that higher scores on each of the displayed measures represent greater severity/impairment.

depression were associated with partially dissociable resting intracranial EEG correlates. To this end, well-validated measures of neuroticism (NEO), reward learning (PRT), and cognitive control (Eriksen Flanker Task) were administered to a sample of depressed adults and correlated with source-localized estimates of resting EEG activity.

Interestingly, the majority of findings emerged in the gamma band. Of note, a combined EEG/FDG-PET study (Oakes et al, 2004) revealed that, of all the classic EEG bands, positive correlations between LORETA estimates and resting brain metabolic activity were strongest for gamma. Accordingly, we interpret increased levels of gamma current density as reflecting increased resting brain activity. Here, neuroticism was associated with increased resting gamma current density in a single cluster localized to the OFC and ventral portions of the ACC (ie, subgenual BA25 and rostral BA24). The latter ACC finding partially confirmed our hypotheses. These ventral ACC regions correspond to the 'affective' subdivision of the ACC, which is densely interconnected with limbic and paralimbic structures (eg, amygdala, nucleus accumbens), has been implicated in emotion expression/ regulation, and is a critical hub in the default mode network linked to self-referential processing (Buckner et al, 2008; Etkin et al, 2011). Previous fMRI studies have associated elevated neuroticism with ACC activity (Haas et al, 2007; Servaas et al, 2013). One study (Haas et al, 2007) observed that greater neuroticism correlated with elevated subgenual ACC activation in a nonclinical sample engaged in an emotional conflict task. Our findings extend these reports by showing that neuroticism was associated with elevated subgenual and ventral ACC gamma activity during rest among an unmedicated MDD sample, suggesting that depressed subjects with elevated neuroticism might engage in maladaptive self-referential processing.

Studies probing the neural substrates of neuroticism must contend with potential confounding variables, including current depressive symptoms and related personality variables. Indeed, neuroticism has been repeatedly shown to covary with current depressive symptoms (Klein et al, 2011), which, in turn, have been associated with hyperactivity in ventral ACC regions (Gotlib et al, 2005; Siegle et al, 2007). In an effort to disentangle trait neuroticism effects from the latter confounds, we controlled for current depressive symptoms and the other four NEO factors. Notably, the
neuroticism-ACC gamma association remained significant, suggesting that it more likely reflects the trait-like aspects of neuroticism, rather than current depressive symptoms or related personality trait confounds.

The neuroticism-OFC association was not anticipated. However, neuroticism has previously been linked to both reduced cortical thickness (Rauch et al, 2005; Wright et al, 2006) and gray matter volume (Kringelbach and Rolls, 2004) in the OFC. Moreover, in addition to its role in value encoding, the OFC has been implicated in emotional processing and regulation/expression (Goodkind et al, 2012; Rolls and Grabenhorst, 2008). In spite of parallels with prior reports, the current link between OFC function and neuroticism awaits replication.

Our hypotheses regarding reward learning were partially supported. Specifically, blunted reward learning was associated with reduced gamma activity in the left dlPFC and OFC. Highlighting specificity, these associations remained significant when controlling for current depressive symptoms and discriminability (an index of task difficulty). Interestingly, gamma OFC also emerged as a significant predictor of participants' sensitivity to rewards, as derived from computational modeling of trial-by-trial PRT performance. Prior research has implicated both the OFC (Hornak et al, 2004; O'Doherty, 2004) and left dlPFC (Ahn et al, 2013; Pizzagalli et al, 2005b) in reward learning. In a prior study using a different task, blunted reward responsiveness was associated with reduced resting OFC and left dlPFC activity among healthy controls (Pizzagalli et al, 2005b). However, in contrast to the present study, the latter findings emerged in the alpha2, rather than gamma, frequency band. More recent research has implicated gamma activity in reward processing (Marco-Pallarés et al, 2015). Contrary to our hypotheses, resting dACC activity was not associated with reward learning across any EEG band examined.

With regards to cognitive control, greater Flanker interference accuracy effects were associated with reduced left dlPFC gamma power. When decomposing this effect, we found that reduced left dlPFC gamma correlated with lower accuracy for incongruent $(r=0.35, p=0.001)$, but not congruent $(r=0.08, p=0.440)$, trials. [Although these findings highlight specificity, it is important to emphasize that variance in accuracy was reduced for the congruent $(\mathrm{SD}=$ $0.02)$, relative to the incongruent $(S D=0.13)$, trials, which 
may have limited the ability to detect a significant association between LORETA current density and accuracy on congruent trials.] Similarly, left dlPFC gamma activity was negatively associated with interference RT effects. The dlPFC has been strongly implicated in cognitive control (Pizzagalli, 2011; Ridderinkhof et al, 2004) and previous studies have linked the left, but not right, dlPFC with cognitive control (MacDonald et al, 2000), including studies using the Flanker task (Fassbender et al, 2006). In contrast to these findings from task-related fMRI paradigms, the current results indicate that greater resting left dlPFC activity predicts performance on a cognitive control task. Within our ROIbut not whole-brain-analyses, increased alpha2 activity in the right dlPFC and decreased theta and alpha2 activity in the dACC were each associated with greater interference RT effects. Because the theta and alpha2 effects were not confirmed in whole-brain analyses, replications are warranted. Importantly, most findings remained significant when controlling for current depressive symptoms and the other endophenotypes.

With regards to our gamma findings, there is evidence that gamma oscillations may facilitate cortical circuit performance and enhance information transmission by increasing signalto-noise ratio within neocortical circuits (Sohal et al, 2009). Although speculative, it is possible that the latter mechanism partially explains the observed findings (eg, greater gamma activity in the left dlPFC reflects relatively enhanced cortical circuit performance in this region, which in turn predicts improved cognitive control performance on the Flanker).

The candidate endophenotypes examined in the current study are themselves complex and multifaceted constructs, which can be parsed into subcomponents. For example, neuroticism has been defined with terms ranging from temperamental, worrying, insecure, and self-conscious (although most, if not all, personality theorists agree that negative emotionality is a defining component; McCrae and Costa, 1987). Similarly, cognitive control is an umbrella term encompassing a range of related abilities, including the ability to inhibit automatic or prepotent responses, shift in a flexible manner between tasks or mental sets, and update working memory in order to retain only relevant information (Friedman et al, 2008; Snyder, 2013). Thus, fruitful findings may emerge from studies examining whether different subcomponents of promising endophenotypes have at least partially dissociable neurobiological signatures.

It is also important to highlight that these endophenotypes are not unique to MDD, but rather cut across several DSM diagnostic categories. Thus, it will be important to investigate the transdiagnostic value of candidate endophenotypes by examining their neurobiological and genetic substrates across psychiatrically heterogeneous samples. The current study exclusively focused on MDD given that we were specifically interested in exploring whether the welldocumented heterogeneity in this disorder category mapped onto, at least partially, dissociable neural circuitry.

The extent to which these candidate endophenotypes have diagnostic or clinical utility remains an open question. With regards to the present data, an exploratory cluster analysis indicated that three subgroups of depressed patients could be differentiated on the basis of endophenotype profiles, including a subgroup with relatively elevated neuroticism, another with greater impairments in cognitive control, and a final group with relatively blunted reward learning (Figure 4). Although speculative, knowledge of endophenotype profiles may ultimately help inform optimal treatment selection. For example, depressed individuals with impaired cognitive control may benefit from interventions with procognitive effects (eg, vortioxetine, Mahableshwarkar et al, 2015). Similarly, certain pharmacological (eg, bupropion) and psychotherapeutic (eg, behavioral activation; Dichter et al, 2009) interventions may be particularly beneficial for those depressed individuals characterized by blunted reward learning and reward-circuitry deficits.

\section{Limitations}

Several limitations of the present study should be noted. First, EEG source localization techniques cannot adequately probe subcortical structures (eg, amygdala, caudate). Although EEG has the benefits of being non-invasive and relatively inexpensive, and thus may be relatively more practical as a diagnostic tool in future clinical settings, it will be important for subsequent studies examining the neural correlates of plausible MDD endophenotypes to employ modalities with greater spatial resolution (eg, fMRI), as well as the ability to probe networks (eg, DMN, salience network). Second, our findings were primarily in the gamma band. There is some evidence suggesting that current density in higher bands (eg, gamma and beta) may be contaminated by muscle or EMG artifacts (Whitham et al, 2007). Although the influence of muscle activity cannot be ruled out, we believe it is unlikely that the current gamma findings (which emerged from resting, task-free EEG data) are due to muscle activity owing to their specificity in terms of hypothesized hemispheric laterality and conditions. Finally, nicotine dependence was not exclusionary, which may represent a possible confound (eg, due to nicotine's possible effects on cognitive and reward function).

\section{FUNDING AND DISCLOSURE}

The EMBARC study was supported by the National Institute of Mental Health of the National Institutes of Health under award numbers U01MH092221 (Trivedi, M.H.) and U01MH092250 (McGrath, P.J., Parsey, R.V., Weissman, M. M.). The content is solely the responsibility of the authors and does not necessarily represent the official views of the National Institutes of Health. Drs. Webb, Dillon and Pizzagalli were supported by NIMH F32 MH099801, R00 MH094438, and R01 MH068376, respectively. Valeant Pharmaceuticals donated the Wellbutrin XL used in the study. This work was supported by the EMBARC National Coordinating Center at UT Southwestern Medical Center to Madhukar H. Trivedi, M.D., Coordinating PI, and the Data Center at Columbia and Stony Brook Universities. Dr Kurian has received grant support from the following additional sources: Targacept, Inc.; Pfizer, Inc.; Johnson \& Johnson; Evotec; Rexahn; Naurex; and Forest Pharmaceuticals. Dr Trombello owns Merck and J\&J stock. Dr Trivedi is or has been an advisor/consultant to: Abbott Laboratories, Inc., Abdi Ibrahim, Akzo (Organon Pharmaceuticals Inc.), Alkermes, AstraZeneca, Axon Advisors, BristolMyers Squibb Company, Cephalon, Inc., Cerecor, Concert Pharmaceuticals, Inc., Eli Lilly \& Company, Evotec, Fabre Kramer Pharmaceuticals, Inc., Forest Pharmaceuticals, 
GlaxoSmithKline, Janssen Global Services, LLC, Janssen Pharmaceutica Products, LP, Johnson \& Johnson PRD, Libby, Lundbeck, Meade Johnson, MedAvante, Medtronic, Merck, Mitsubishi Tanabe Pharma Development America, Inc., Naurex, Neuronetics, Otsuka Pharmaceuticals, Pamlab, ParkeDavis Pharmaceuticals, Inc., Pfizer Inc., PgxHealth, Phoenix Marketing Solutions, Rexahn Pharmaceuticals, Ridge Diagnostics, Roche Products Ltd., Sepracor, SHIRE Development, Sierra, SK Life and Science, Sunovion, Takeda, Tal Medical/Puretech Venture, Targacept, Transcept, VantagePoint, Vivus, and Wyeth-Ayerst Laboratories. In addition, he has received research support from: Agency for Healthcare Research and Quality (AHRQ), Corcept Therapeutics, Inc., Cyberonics, Inc., National Alliance for Research in Schizophrenia and Depression, National Institute of Mental Health, National Institute on Drug Abuse, Novartis, Pharmacia \& Upjohn, Predix Pharmaceuticals (Epix), and Solvay Pharmaceuticals, Inc. For a comprehensive list of lifetime disclosures of Dr Fava, see http://mghcme.org/faculty/facultydetail/maurizio_fava. Over the past 3 years, Dr McGrath has received research support from the National Institute of Mental Health, the New York State Department of Mental Hygiene, the Research Foundation for Mental Hygiene (New York State), F. Hoffman-LaRoche, Ltd., Naurex Pharmaceuticals, Forest Research and Sunovion Pharmaceuticals. In the past 3 years, Dr Weissman received funding from the National Institute of Mental Health, the National Institute on Drug Abuse, the National Alliance for Research on Schizophrenia and Depression, the Sackler Foundation, and the Templeton Foundation; and received royalties from the Oxford University Press, Perseus Press, the American Psychiatric Association Press and MultiHealth Systems. Dr Weissman declares that none of these present a conflict of interest. Dr Grannemann declares that, except for income received from a primary employer and the above grant (U01MH092221), no financial support or compensation has been received from any individual or corporate entity over the past 3 years for research or professional service and there are no personal financial holdings that could be perceived as constituting a potential conflict of interest. Over the past 3 years, Dr Pizzagalli has received honoraria/consulting fees from Advanced Neuro Technology North America, AstraZeneca, Otsuka America Pharmaceutical, Pfizer, and Servier for activities unrelated to this project. The remaining authors declare no conflict of interest.

\section{ACKNOWLEDGMENTS}

We thank Alex Shackman, $\mathrm{PhD}$, for providing regions-ofinterest definition based on anatomical atlases and landmarks. We also thank Steven Lowen, $\mathrm{PhD}$, and Garrett Fitzmaurice, ScD, for their statistical consultation.

\section{REFERENCES}

Ahn HM, Kim SE, Kim SH (2013). The effects of high-frequency rTMS over the left dorsolateral prefrontal cortex on reward responsiveness. Brain Stimulat 6: 310-314.

Birley AJ, Gillespie NA, Heath AC, Sullivan PF, Boomsma DI, Martin NG (2006). Heritability and nineteen-year stability of long and short EPQ-R Neuroticism scales. Personal Individ Differ 40: 737-747.
Bogdan R, Pizzagalli DA (2009). The heritability of hedonic capacity and perceived stress: a twin study evaluation of candidate depressive phenotypes. Psychol Med 39: 211-218.

Buckner RL, Andrews-Hanna JR, Schacter DL (2008). The Brain's default network. Ann NY Acad Sci 1124: 1-38.

Chan RCK, Gottesman II (2008). Neurological soft signs as candidate endophenotypes for schizophrenia: A shooting star or a Northern star? Neurosci Biobehav Rev 32: 957-971.

Chiu T, Fang D, Chen J, Wang Y, Jeris C (2001). A robust and scalable clustering algorithm for mixed type attributes in large database environment. Proc Seventh ACM SIGKDD Int Conf Knowl Discov Data Min 263-268.

Christensen MV, Kyvik KO, Kessing LV (2006). Cognitive function in unaffected twins discordant for affective disorder. Psychol Med 36: 1119-1129.

Costa PT, Bagby RM, Herbst JH, McCrae RR (2005). Personality self-reports are concurrently reliable and valid during acute depressive episodes. J Affect Disord 89: 45-55.

Dichter GS, Felder JN, Petty C, Bizzell J, Ernst M, Smoski MJ (2009). The effects of psychotherapy on neural responses to rewards in major depression. Biol Psychiatry 66: 886-897.

Eriksen BA, Eriksen CW (1974). Effects of noise letters upon the identification of a target letter in a nonsearch task. Percept Psychophys 16: 143-149.

Etkin A, Egner T, Kalisch R (2011). Emotional processing in anterior cingulate and medial prefrontal cortex. Trends Cogn Sci 15: 85-93.

Farmer A, Redman K, Harris T, Mahmood A, Sadler S, Pickering A et al (2002). Neuroticism, extraversion, life events and depression. The Cardiff Depression Study. Br J Psychiatry 181: 118-122.

Fassbender C, Foxe JJ, Garavan H (2006). Mapping the functional anatomy of task preparation: Priming task-appropriate brain networks. Hum Brain Mapp 27: 819-827.

Fava GA, Guidi J, Porcelli P, Rafanelli C, Bellomo A, Grandi S et al (2012). A cluster analysis-derived classification of psychological distress and illness behavior in the medically ill. Psychol Med 42: 401-407.

Friedman NP, Miyake A, Young SE, DeFries JC, Corley RP, Hewitt JK (2008). Individual differences in executive functions are almost entirely genetic in origin. J Exp Psychol Gen 137: 201-225.

De Fruyt F, Leeuwen K, Van, Bagby RM, Rolland J-P, Rouillon F (2006). Assessing and interpreting personality change and continuity in patients treated for major depression. Psychol Assess 18: 71-80.

Goldstein BL, Klein DN (2014). A review of selected candidate endophenotypes for depression. Clin Psychol Rev 34: 417-427.

Goodkind MS, Sollberger M, Gyurak A, Rosen HJ, Rankin KP, Miller B et al (2012). Tracking emotional valence: the role of the orbitofrontal cortex. Hum Brain Mapp 33: 753-762.

Gotlib IH, Sivers H, Gabrieli JDE, Whitfield-Gabrieli S, Goldin P, Minor KL et al (2005). Subgenual anterior cingulate activation to valenced emotional stimuli in major depression. Neuroreport 16: $1731-1734$.

Gottesman II, Gould TD (2003). The endophenotype concept in psychiatry: etymology and strategic intentions. Am J Psychiatry 160: 636-645.

Haas BW, Omura K, Constable RT, Canli T (2007). Emotional conflict and neuroticism: Personality-dependent activation in the amygdala and subgenual anterior cingulate. Behav Neurosci 121: 249-256.

Hamilton M (1960). A rating scale for depression. I Neurol Neurosurg Psychiatry 23: 56-62.

Holmes AJ, Bogdan R, Pizzagalli DA (2010). Serotonin transporter genotype and action monitoring dysfunction: a possible substrate underlying increased vulnerability to depression. Neuropsychopharmacology 35: 1186-1197.

Hornak J, O'doherty J, Bramham J, Rolls ET, Morris RG, Bullock PR et al (2004). Reward-related reversal learning after 
surgical excisions in orbito-frontal or dorsolateral prefrontal cortex in humans. J Cogn Neurosci 16: 463-478.

Huys QJ, Pizzagalli DA, Bogdan R, Dayan P (2013). Mapping anhedonia onto reinforcement learning: a behavioural metaanalysis. Biol Mood Anxiety Disord 3: 12.

Kendler K, Gatz M, Gardner CO, Pedersen NL (2006). Personality and major depression: A swedish longitudinal, population-based twin study. Arch Gen Psychiatry 63: 1113-1120.

Klein DN, Kotov R, Bufferd SJ (2011). Personality and depression: explanatory models and review of the evidence. Annu Rev Clin Psychol 7: 269-295.

Kringelbach ML, Rolls ET (2004). The functional neuroanatomy of the human orbitofrontal cortex: evidence from neuroimaging and neuropsychology. Prog Neurobiol 72: 341-372.

MacDonald AW, Cohen JD, Stenger VA, Carter CS (2000). Dissociating the role of the dorsolateral prefrontal and anterior cingulate cortex in cognitive control. Science 288: 1835-1838.

Mahableshwarkar AR, Zajecka J, Jacobson W, Chen Y, Keefe RS (2015). A randomized, placebo-controlled, active-reference, double-blind, flexible-dose study of the efficacy of vortioxetine on cognitive function in major depressive disorder. Neuropsychopharmacology 40: 2025-2037.

Marco-Pallarés J, Münte TF, Rodríguez-Fornells A (2015). The role of high-frequency oscillatory activity in reward processing and learning. Neurosci Biobehav Rev 49: 1-7.

McCrae RR, Costa PT (1987). Validation of the five-factor model of personality across instruments and observers. J Pers Soc Psychol 52: 81-90.

McCrae RR, Costa PT (2010). NEO Inventories for the NEO Personality Inventory-3 (NEO-PI-3), NEO Five-Factor Inventory3 (NEO-FFI-3), NEO Personality Inventory-Revised (NEO PI-R): Professional Manual. PAR: Lutz, FL.

Meng X, Rosenthal R, Rubin DB (1992). Comparing correlated correlation coefficients. Psychol Bull 111: 172-175.

Modell S, Huber J, Holsboer F, Lauer CJ (2003). The Munich Vulnerability Study on Affective Disorders: risk factors for unipolarity versus bipolarity. J Affect Disord 74: 173-184.

Oakes TR, Pizzagalli DA, Hendrick AM, Horras KA, Larson CL, Abercrombie HC et al (2004). Functional coupling of simultaneous electrical and metabolic activity in the human brain. Hum Brain Mapp 21: 257-270.

O'Doherty JP (2004). Reward representations and reward-related learning in the human brain: insights from neuroimaging. Curr Opin Neurobiol 14: 769-776.

Ouimette PC, Klein DN, Pepper CM (1996). Personality traits in the first degree relatives of outpatients with depressive disorders. J Affect Disord 39: 43-53.

Pascual-Marqui RD, Lehmann D, Koenig T, Kochi K, Merlo MCG, Hell D et al (1999). Low resolution brain electromagnetic tomography (LORETA) functional imaging in acute, neuroleptic-naive, first-episode, productive schizophrenia. Psychiatry Res Neuroimaging 90: 169-179.

Pechtel P, Dutra SJ, Goetz EL, Pizzagalli DA (2013). Blunted reward responsiveness in remitted depression. J Psychiatr Res 47: 1864-1869.

Pizzagalli DA (2011). Frontocingulate dysfunction in depression: toward biomarkers of treatment response. Neuropsychopharmacology 36: 183-206.

Pizzagalli DA (2014). Depression, stress, and anhedonia: toward a synthesis and integrated model. Annu Rev Clin Psychol 10: 393-423.

Pizzagalli DA, Evins AE, Schetter EC, Frank MJ, Pajtas PE, Santesso DL et al (2008). Single dose of a dopamine agonist impairs reinforcement learning in humans: Behavioral evidence from a laboratory-based measure of reward responsiveness. Psychopharmacology (Berl) 196: 221-232.
Pizzagalli DA, Jahn AL, O’Shea JP (2005a). Toward an objective characterization of an anhedonic phenotype: a signal-detection approach. Biol Psychiatry 57: 319-327.

Pizzagalli DA, Sherwood RJ, Henriques JB, Davidson RJ (2005b). Frontal brain asymmetry and reward responsiveness a sourcelocalization study. Psychol Sci 16: 805-813.

Rauch SL, Milad MR, Orr SP, Quinn BT, Fischl B, Pitman RK (2005). Orbitofrontal thickness, retention of fear extinction, and extraversion. Neuroreport 16: 1909-1912.

Ridderinkhof KR, van den, Wildenberg WPM, Segalowitz SJ, Carter CS (2004). Neurocognitive mechanisms of cognitive control: the role of prefrontal cortex in action selection, response inhibition, performance monitoring, and reward-based learning. Brain Cogn 56: 129-140.

Rolls ET, Grabenhorst F (2008). The orbitofrontal cortex and beyond: from affect to decision-making. Prog Neurobiol 86: 216-244.

Rush AJ, Trivedi MH, Ibrahim HM, Carmody TJ, Arnow B, Klein DN et al (2003). The 16-Item quick inventory of depressive symptomatology (QIDS), clinician rating (QIDS-C), and selfreport (QIDS-SR): a psychometric evaluation in patients with chronic major depression. Biol Psychiatry 54: 573-583.

Rushworth MF, Buckley MJ, Behrens TE, Walton ME, Bannerman DM (2007). Functional organization of the medial frontal cortex. Curr Opin Neurobiol 17: 220-227.

Santesso DL, Dillon DG, Birk JL, Holmes AJ, Goetz E, Bogdan R et al (2008). Individual differences in reinforcement learning: behavioral, electrophysiological, and neuroimaging correlates. NeuroImage 42: 807-816.

Sarapas C, Shankman SA, Harrow M, Goldberg JF (2012). Parsing trait and state effects of depression severity on neurocognition: evidence from a 26-year longitudinal study. J Abnorm Psychol 121: $830-837$.

Servaas MN, Velde J, van der, Costafreda SG, Horton P, Ormel J, Riese $\mathrm{H}$ et al (2013). Neuroticism and the brain: a quantitative meta-analysis of neuroimaging studies investigating emotion processing. Neurosci Biobehav Rev 37: 1518-1529.

Siegle G, Ghinassi F, Thase M (2007). Neurobehavioral therapies in the 21st century: summary of an emerging field and an extended example of cognitive control training for depression. Cogn Ther Res 31: 235-262.

Snyder HR (2013). Major depressive disorder is associated with broad impairments on neuropsychological measures of executive function: a meta-analysis and review. Psychol Bull 139: 81-132.

Sohal VS, Zhang F, Yizhar O, Deisseroth K (2009). Parvalbumin neurons and gamma rhythms enhance cortical circuit performance. Nature 459: 698-702.

Stins JF, Baal GCM, van, Polderman TJ, Verhulst FC, Boomsma DI (2004). Heritability of Stroop and flanker performance in 12-year old children. BMC Neurosci 5: 49.

Whitham EM, Pope KJ, Fitzgibbon SP, Lewis T, Clark CR, Loveless S et al (2007). Scalp electrical recording during paralysis: quantitative evidence that EEG frequencies above $20 \mathrm{~Hz}$ are contaminated by EMG. Clin Neurophysiol 118: 1877-1888.

Winkler AM, Ridgway GR, Webster MA, Smith SM, Nichols TE (2014). Permutation inference for the general linear model. NeuroImage 92: 381-397.

Wöstmann NM, Aichert DS, Costa A, Rubia K, Möller H-J, Ettinger U (2013). Reliability and plasticity of response inhibition and interference control. Brain Cogn 81: 82-94.

Wright CI, Williams D, Feczko E, Barrett LF, Dickerson BC, Schwartz CE et al (2006). Neuroanatomical correlates of extraversion and neuroticism. Cereb Cortex 16: 1809-1819. 